\title{
Voxel-based morphometry of adulthood patients with temporal lobe epilepsy
}

\author{
A Labate ${ }^{1,2^{*}}$, A Gambardella ${ }^{1,2}$, U Aguglia', L Mumoli ${ }^{1}$, G Palamara' ${ }^{1}$, A Fratto ${ }^{1}$, M Sturniolo ${ }^{2}$, A Quattrone ${ }^{1,2}$ \\ From de Senectute: Age and Health Forum \\ Catanzaro, Italy. 5-7 December 2009
}

\section{Background}

Partial epilepsy represents almost $70 \%$ of the epileptic syndrome. It is characterized by partial seizures that arise from the restricted area of the cerebral cortex. Temporal lobe epilepsy (TLE) is the most frequent form of cryptogenic partial epilepsies [1]. The mesial temporal sclerosis is the pathologic abnormality most frequently detected in post mortem studies of subjects with TLE. Voxel-based morphometry (VBM) is a new MRI tool that has been developed to assess differences of brain tissue volumes between subject groups and has been applied in various studies of patients with TLE [2]. In refractory TLE (rTLE) gray (GM) and white (WM) matter abnormalities are not confined to the hippocampus but also are found in extrahippocampal structures [3]. Less is known about mild TLE (mTLE). In the present work, we used optimized voxel-based morphometry (VBM) to identify GM abnormalities beyond the hippocampus in rTLE and in MTLE with evidence of hippocampal sclerosis (HS).

\section{Materials and methods}

Brain MRI and VBM of GM with modulation was performed on 30 unrelated patients with mTLE (56\% women; mean age $35.6+15.2$ years), 19 patients with rTLE (52\% women; mean age $38.4+17.4$ years) and 37 healthy controls ( 25 women, mean age $37.3+10.6$ years). MRI diagnosis of MTS was based on the atrophy of the hippocampal formation and/or mesial temporal hyperintensity on FLAIR or T2 images, or both.

\section{Results}

All patients (rTLE and mTLE) did not have any generalised tonic-clonic seizures for at least three weeks before the scanning. Respectively, mTLE patients showed GM volume reduction of the bilateral thalamus and left hippocampus (FWE < 0.05) whereas rTLE in the thalamus bilaterally (FWE $<0.05)$ when compared with controls. Conversely, no differences of GM concentrations were found between rTLE and mTLE.

\section{Conclusions}

In either rTLE and mTLE, VBM shows GM reductions not confined to the hippocampus but mainly in the thalamus bilaterally. Moreover, no GM differences were found between the two groups. This supports the hypothesis that mTLE and rTLE might lie along a biological continuum.

\section{Author details}

${ }^{1}$ Institute Neurology, University Magna Græcia, Catanzaro, Italy. ${ }^{2}$ Institute of Neurological Sciences, National Research Council, Piano Lago, Mangone, Cosenza, Italy.

\section{Published: 19 May 2010}

\section{References}

1. Berkovic SF, McIntosh A, Howell RA, et al: Familial temporal lobe epilepsy: a common disorder identified in twins. Ann Neurol 1996, 40:227-235.

2. Labate A, Cerasa A, Gambardella A, et al: Hippocampal and thalamic atrophy in mild temporal lobe epilepsy: a VBM study. Neurology. 2008, 71(14):1094-101.

3. Keller SS, Roberts $\mathrm{N}$ : Voxel-based morphometry of temporal lobe epilepsy: an introduction and review of the literature. Epilepsia 2008, 49(5):741-57.

doi:10.1186/1471-2318-10-S1-A95

Cite this article as: Labate et al: Voxel-based morphometry of adulthood patients with temporal lobe epilepsy. BMC Geriatrics 2010 10(Suppl 1):A95.

${ }^{1}$ Institute Neurology, University Magna Græcia, Catanzaro, Italy 